\title{
A RECORDING AND ANALYZING SYSTEM FOR CUTANEOUS ELECTROGASTROGRAPHY
}

\author{
Han-Chang Wu ${ }^{1}$, Student Member, Shao-Cheng Wang ${ }^{1}$, Shuenn-Tsong Young ${ }^{2}$, and Te-Son Kuo ${ }^{1}$ \\ ${ }^{1}$ Department of Electrical Engineering, National Taiwan University \\ ${ }^{2}$ Institute of Biomedical Engineering, National Yang-Ming University
}

\section{E-mail:wuman@tpts1.seed.net.tw}

\begin{abstract}
The recording of gastric activities by surface electrodes is called electrogastrography(EGG). It should be carefully filtered and amplified because of its ultra-low frequency, tiny and noisy signal. A new system, including a signal acquisition device and Windows-based software, was designed to record and analyze the EGG signal. Many volunteers and patients have been successfully tested by the system.
\end{abstract}

\section{Introduction}

Gastric activities were first measured cutaneously in 1922 and this physiological signal is often called electrogastrography(EGG)[1]. It is more and more important and attractive because of its non-invasive measurement. However, the EGG signal is hard to be measured because of (1) low frequency: about 3 cycles per minutes (cpm) for normal people, 1 2 cpm for bradygastria condition and $6 \sim 9$ cpm for tachygastria; (2) tiny signal level about 50 500uV; (3) noisy: the EGG signal is measured by surface electrodes on human upper abdomen[2]. It is easily coupled by other physiological signals like EKG or respiration. Therefore, the EGG must be carefully filtered and amplified therefore high SNR signal could be achieved. The EGG signal is not considered useful by time domain waveform. Some parameters are used to extract more information from the EGG including amplitude and frequency. $[3,4,5]$

A complete and easy-to-use system is designed and tested in hospitals to record the EGG signal and to analyze it by some defined parameters. The system includes a signal acquisition device to amplify, filter, and digitize the signal. We have made a printed circuit board (PCB) for enhanced signal quality. An analyzing software assesses the digitized data and then analyzes it based on short term Fourier transform (STFT) and parameters are figured out. This software is executed on the Windows-95 operating system as a more friendly user interface.

\section{Methods}

The system consists of an external signal acquisition device and a host PC running our software, as shown in fig. 1. The signal acquisition device measured the EGG signal from electrodes on upper abdomen. After amplifying and filtering the EGG signal, a 12-bit analog to digital converter (ADC) was used to digitize the EGG signal, and then transferred the data to host with defined protocol through RS-232 serial port interface.

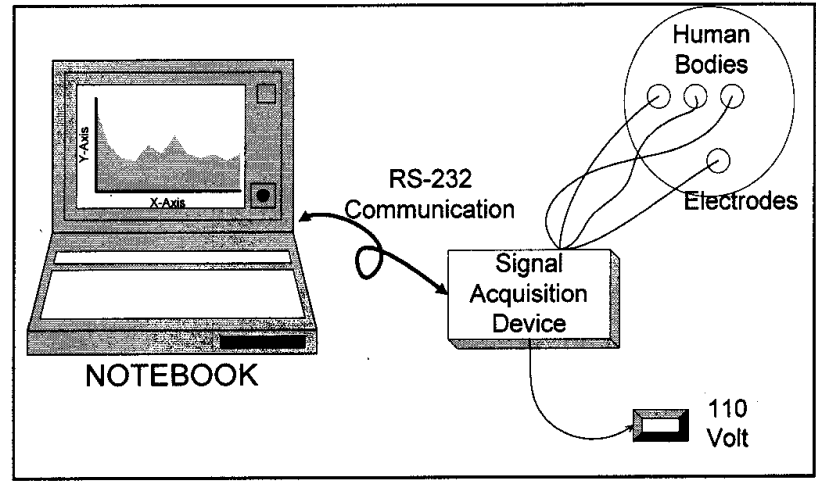

Fig.1. System Configuration

a. Signal Acquisition Device:

The hardware block diagram of the signal acquisition device is shown on fig. 2. An AT89C52 microprocessor was used to collect data and communicate with the host. We used four EKG electrodes to access the EGG signals ( 3 for signals and 1 for the reference) from three different channels. The recorded EGG signal was first pre-amplified by an instrumental amplifier to match the high impedance of human surface. A 4-th order active high pass filter with $0.01 \mathrm{~Hz}$ cutoff frequency and a 4-th order active low pass filter with 0.5 $\mathrm{Hz}$ cut-off frequency were used to filter out other noisy signals like EKG, respiration, and motion artifacts. Then a high-gain amplifier was used to provide the total gain of the system with 4000 40000. Isolation amplifiers were used after the high-gain amplifier for safety consideration. A $0.5 \mathrm{~Hz}$ precision clock source was selected to provide stable $1 \mathrm{~Hz}$ sampling rate. The microprocessor was interrupted by the clock. An analog multiplexer was used to select one of three channels of the EGG signals and then the microprocessor got the digital data from the ADC. Every second the microprocessor sent the EGG data to the host via RS-232 interface with a pre-defined data format to provide expansion capability and data integrity.

The signal acquisition device was powered by $12 \mathrm{~V} \mathrm{DC}$ voltage from a commercial regulated DC adapter. To achieve 
system requirements, five different power levels were provided by the power circuit. For human safety, two isolated $+8 \mathrm{~V}$ and $-8 \mathrm{~V}$ DC voltages were provided by a isolation power transformer. Besides, two non-isolated $+8 \mathrm{~V}$ and $-8 \mathrm{~V}$ DC voltages were regulated by common LM7808 and LM7908 linear regulators. A 5V DC voltage was also used to provide the power of digital circuit.

\section{b. Analyzing Software}

The host software was programmed with "Borland $\mathrm{C}+\mathrm{H}$ Builder V1.0 Professional" on Windows $95^{\mathrm{TM}}$ platform, as shown in Fig.3. The host software received data from the signal acquisition device and then decoded the data into EGG signal. The host software contained three portions, time domain waveform, spectrum and parameters. The EGG signal could be real-time displayed and the parameters would be figured out at the same time. The EGG signal, processed data, and historical information can be saved in a signal file and then could be replayed by the same software.

\section{Results}

The system has been successfully tested by both simulation of a signal generator and volunteers. We used a signal with $1.15 \mathrm{mV}$ level and $3 \mathrm{cpm}$ frequency to verify the performance of our system. Its spectrum is shown in Fig.3. The signal-to-noise ratio is around $50 \mathrm{~dB}$. The PCB size is 7' $\mathrm{L}$ and 5.8"W packaged in a 8.3 " $\mathrm{L}, 6.5$ "W and 1.6 "H box. The view of the software is shown in fig.4. Besides the time domain waveform, it also provides pseudo 3D spectrum. Parameters are figured out and shown by the software. The software also provides historical data storage and printing functions.

\section{Conclusion}

In this study we presented a complete system for recording and analyzing the EGG signal. A small but high performance and safe device was used to carefully assess the raw EGG signal. A user friendly analyzing software was executed under windows platform. We used a notebook to run the software and therefore the whole system could be brought easily. It is much more convenient to acquire the EGG research than before, no matter in the laboratory or in the hospital.

\section{Acknowledgement}

Special thanks to Full-Young Chang, M.D., Chief of GI Division, VGH- Taipei, for his support of EGG experiments.

\section{Reference}

1. Alvarez WC. The electrogastrogram and what is shows. JAMA 1922;78:1116-1119.

2. Chen J, McCallum RW. Electrogastrography: Measurement, analysis and prospective applications. Med Biol Eng Comput 1991;29:339-50.

3. Chen J, Vandewalle J, Sansen W, et al. Adaptive spectral analysis of cutaneous electrogastric signals using autoregressive moving average modelling. Med Biol Eng Comput 1990;28:531-6

4. Chen J, Vandewalle J, Sansen W, et al. Multichannel Adaptive Enhancement of the Electrogastrography. IEEE Tran. on Bio. Eng. 1990;17:285-94

5. Chen J, Vandewalle J, Sansen W, et al. Adaptive method for cancellation of respiratory artifact in electrogastric measurements. Med Biol Eng Comput 1989;27:57-63

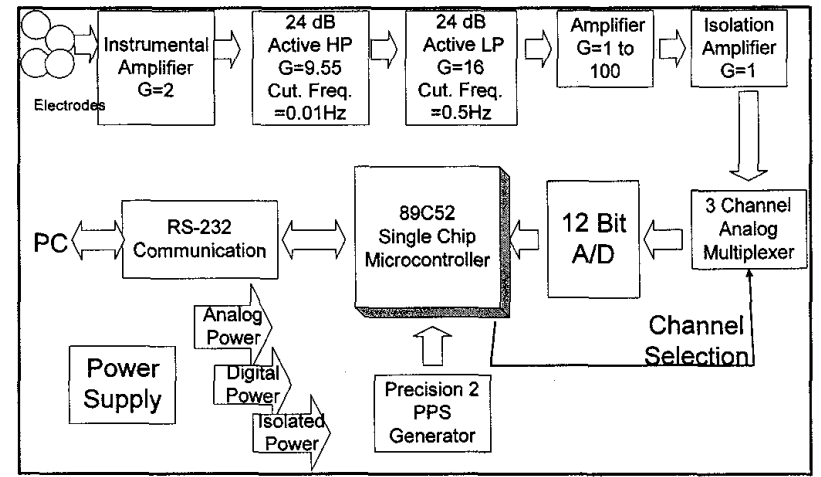

Fig.2. Hardware Block Diagram

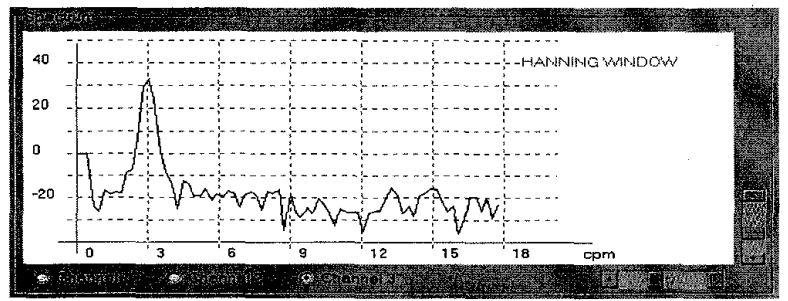

Fig.3. System Performance

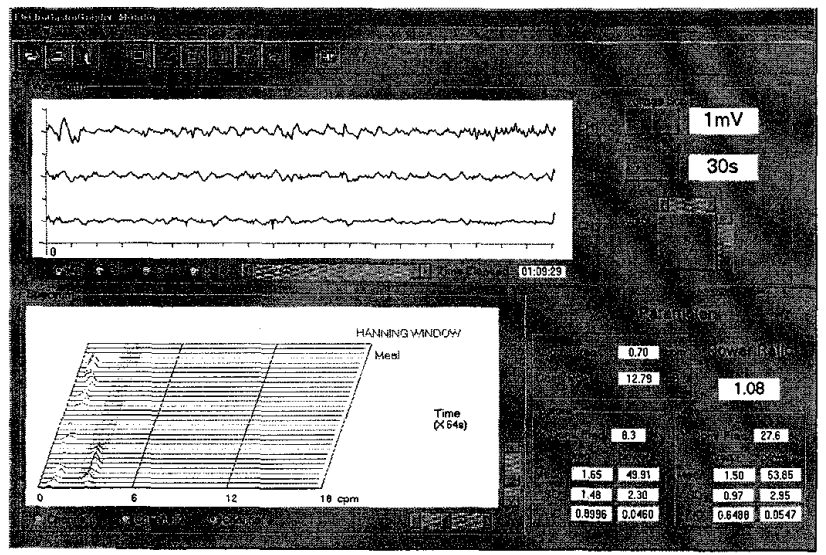

Fig.4. Software Overview 